\title{
Mechanistic basis and therapeutic relevance of hypocalcemia during severe COVID-19 infection
}

\author{
Vijay P. Singh $\mathbb{D}^{1} \cdot$ Biswajit Khatua ${ }^{1} \cdot$ Bara El-Kurdi ${ }^{2} \cdot$ Christopher Rood $^{3}$ \\ Received: 21 August 2020 / Accepted: 14 October 2020 / Published online: 24 October 2020 \\ (c) Springer Science+Business Media, LLC, part of Springer Nature 2020
}

\section{To the Editor:}

We read the article Hypocalcemia is highly prevalent and predicts hospitalization in patients with COVID-19 pandemic by Di Filippo et al. [1] published in the June 2020 issue of Endocrine with great interest. We agree with their observation that hypocalcemia is associated with severe COVID-19. However we would like to make your readers aware of the mechanisms that underlie this acute hypocalcemia and its therapeutic relevance.

It is notable that while the authors cite calcium as being important for viral fusion, this would conflict with their finding of hypocalcemia being associated with severe COVID-19. Moreover, acute hypocalcemia during severe COVID-19 is caused by an interaction of calcium with unsaturated fatty acids released during the disease, and is independent of chronic vitamin D deficiency, or hypoparathyroidism for the reasons mentioned below. This distinction is important, since early correction of hypocalcemia may be a therapeutic target to improve COVID-19 outcomes, and not just a predictor of severity. The salient features linking severe COVID-19 and hypocalcemia are:

1. Patients with severe COVID-19 have elevated unbound fatty acids [2], and unsaturated fatty acids [3].

2. Unsaturated fatty acids can bind calcium with a favorable $(-20 \mathrm{KJ} / \mathrm{mol})$ enthalpy and trigger acute hypocalcemia [4].

3. Ionized calcium levels were measured in this study, which is notable, as calcium interacts with fatty acids when in an ionized state [4].

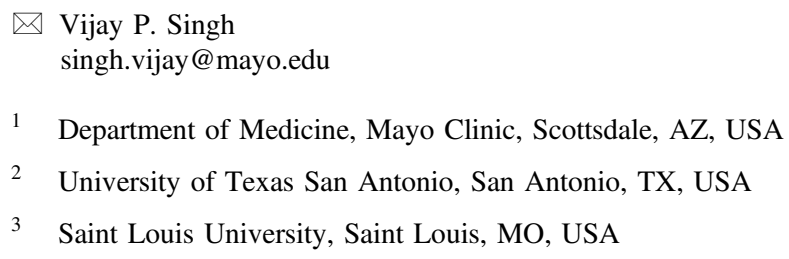

4. Unsaturated fatty acids can induce the cytokine storm [5] and multisystem organ failure [2, 4] such as noted in COVID-19.

5. During severe COVID-19, hypocalcemia is accompanied by hypoalbuminemia [2], and both of these can be induced acutely by unsaturated fatty acids [2], unlike hypoparathyroidism or vitamin D deficiency.

6. Chronic vitamin D deficiency states in adults, such as osteomalacia very rarely present with the acute hypocalcemia seen in COVID-19, but as mild baseline chronic hypocalcemia, irrespective of whether the patient has COVID-19 infection.

7. The "calcium albumin correction" pseudo-normalizes hypocalcemia concurrent with hypoalbuminemia (for e.g., a serum calcium of $5.9 \mathrm{mg} / \mathrm{dl}$ with an albumin of $0.1 \mathrm{gm} / \mathrm{dl}$, to a calcium of $9.0 \mathrm{mg} / \mathrm{dl}$; https: $/ / \mathrm{www}$. mdcalc.com/calcium-correction-hypoalbuminemia), and should not be applied to COVID-19 associated hypocalcemia [2].

8. Lastly, the therapeutic relevance of this unsaturated fatty acid-calcium interaction lies in correction of calcium (and albumin) deficits early in the disease. By binding and neutralizing the unbound unsaturated fatty acids early in the disease [2], such a correction may prevent the mitochondrial injury [6] that results in widespread cell injury, organ failure, and sepsis later during severe COVID-19 [2]. Delayed correction of these deficits after organ failure has set in may be of little help [2].

We hope your readers find the above explanation of acute hypocalcemia during severe COVID-19 to be mechanistically and therapeutically relevant, and realize its value beyond being a prognostic marker.

\section{Compliance with ethical standards}

Conflict of interest The authors declare that they have no conflict of interest. 
Publisher's note Springer Nature remains neutral with regard to jurisdictional claims in published maps and institutional affiliations.

\section{References}

1. L. Di Filippo, et al. Hypocalcemia is highly prevalent and predicts hospitalization in patients with COVID-19. Endocrine 68, 475-478 (2020). https://doi.org/10.1007/s12020-020-02383-5

2. B. El-Kurdi, et al. Mortality from coronavirus disease 2019 increases with unsaturated fat and may be reduced by earlBy calcium and albumin supplementation. Gastroenterology (2020). https://doi.org/10.1053/j.gastro.2020.05.057
3. T. Thomas, et al. COVID-19 infection alters kynurenine and fatty acid metabolism, correlating with IL-6 levels and renal status. JCI Insight 5, (2020). https://doi.org/10.1172/jci. insight. 140327

4. B. Khatua, et al. Ringer's Lactate prevents early organ failure by providing extracellular calcium. J. Clin. Med. 9, (2020). https://doi. org $/ 10.3390 / \mathrm{jcm} 9010263$

5. C. de Oliveira, et al. Pancreatic triglyceride lipase mediates lipotoxic systemic inflammation. J. Clin. Investig. 130, 1931-1947 (2020). https://doi.org/10.1172/JCI132767

6. S. Navina, et al. Lipotoxicity causes multisystem organ failure and exacerbates acute pancreatitis in obesity. Sci. Transl. Med. 3, 107ra110 (2011). https://doi.org/10.1126/scitranslmed.3002573 